\title{
Numerical simulation for entrainment of forced turbulent fountains
}

\author{
L. A. Awin ${ }^{1} \quad$ S. W. Armfield ${ }^{2} \quad$ N. Williamson ${ }^{3}$ \\ M. P. Kirkpatrick $\quad$ W. Lin $^{5}$
}

(Received 25 February 2019; revised 16 June 2019)

\begin{abstract}
Numerical simulations are used to investigate the entrainment for forced turbulent fountains over a range of Reynolds numbers and Froude numbers, with ranges based on the fountain source properties. Other fountain properties such as height and width are also examined to provide information on the general structure of the fountains. The results show that the fountains have minimal Reynolds number dependency, while they have a strong linear relation with the Froude number for the cases considered in this study. The entrainment coefficient is obtained as well as scaling constants for height and width in terms of the Froude number.
\end{abstract}

DOI:10.21914/anziamj.v60i0.14051 gives this article, (c) Austral. Mathematical Soc. 2019. Published August 29, 2019, as part of the Proceedings of the 18th Biennial Computational Techniques and Applications Conference. ISSN 1445-8810. (Print two pages per sheet of paper.) Copies of this article must not be made otherwise available on the internet; instead link directly to the DOI for this article. 


\section{Contents}

1 Introduction

C157

2 Numerical method

C159

3 Results

C161

4 Conclusions

C168

\section{Introduction}

Fountains occur when a dense fluid is projected upwards into a less dense fluid. The upward discharge of the dense fluid is opposed by buoyancy, and further limited by the entrainment from the ambient fluid. The fountain structure is described as a rising fluid surrounded by falling fluid, with a crown region at the top where the rising fluid turns to become falling fluid, as shown in Figure 1(a). Fountains are widely found in nature and industrial applications such as volcanic eruptions, atmospheric convection, building ventilation, refueling fuel tanks, and air conditioning systems, where the overall behaviour of the flow or device are strongly influenced by the fountain properties $[2,6,10]$. In turbulent fountains, fluid entrainment $\alpha$ plays a key role in controlling the properties of the fountain, such as penetration height, width and mixing.

The control parameters of fountains are typically two non-dimensional numbers, the Froude number Fr and the Reynolds number Re, where the former characterises the fountain flow as weak or forced while the latter characterises the flow as laminar or turbulent. For Froude numbers greater than three, the fountain is forced [8] and for Reynolds numbers greater than 2000, the fountain is classified as fully turbulent [16].

Scaling analysis shows that, for forced fountains, the average fountain height $Z_{m}$ 
is expected to scale with the Froude number, $Z_{\mathrm{m}} / R_{\mathrm{o}} \approx \mathrm{CFr}$, where $R_{\mathrm{o}}$ is the source radius and $\mathrm{C}$ is a constant $[2,3,5,8,11,14,16,17]$. This relation has been numerically and experimentally validated by a number of studies over the Froude number range $3 \leqslant \mathrm{Fr} \leqslant 300$, although the 'constant' $\mathrm{C}$ varies from 2.1 to $3.06[2,5,11,14,16]$.

The first fountain models considered only the starting fountain, that is, before the crown and falling flow regions develop, and this fountain was treated as a negatively buoyant jet. It was assumed the entrainment of the ambient fluid into the negatively buoyant jet was the same as that for the neutrally buoyant jet, where the entrainment is defined as the radial velocity normalised by axial velocity. The model predicted a linear Fr dependency for the penetration height $[1,8,12]$. This technique was later extended to estimate the penetration height of fully developed fountains by including a similar entrainment assumption for the ambient fluid to the falling fluid and the falling fluid to the rising fluid [3], with the results validated against the experimental results of [11]. However, numerical results suggested the simple self-similar entrainment relations may not be suitable for fountain flows [15]. Laboratory measurements of total fountain entrainment suggested that in the forced turbulent regime the total entrainment $Q_{E}$ has a linear relation with the Froude number, $\mathrm{Q}_{\mathrm{E}} / \mathrm{Q}_{\mathrm{o}} \approx 0.71 \mathrm{Fr}$, where $\mathrm{Q}_{\mathrm{o}}$ is the fountain source volume flux [4].

The overall behaviour, penetration height, and width of the fountain are strongly dependent on the entrainment of the ambient fluid into the fountain and the mixing within the fountain. As noted above, current models are based on plume and jet type entrainment laws with constant entrainment coefficients. Little direct measurement of the entrainment and internal structure of fountains have been conducted, either experimentally or via direct numerical simulation. In this study a numerical investigation of forced turbulent fountain flow is performed to quantify the entrainment of the ambient fluid into the fountain and to provide scaling relations for the fountain height and width. 
Ambient Fluid

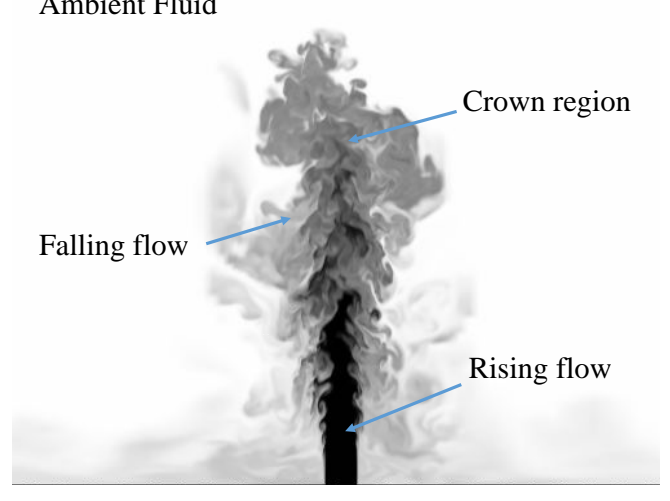

(a)

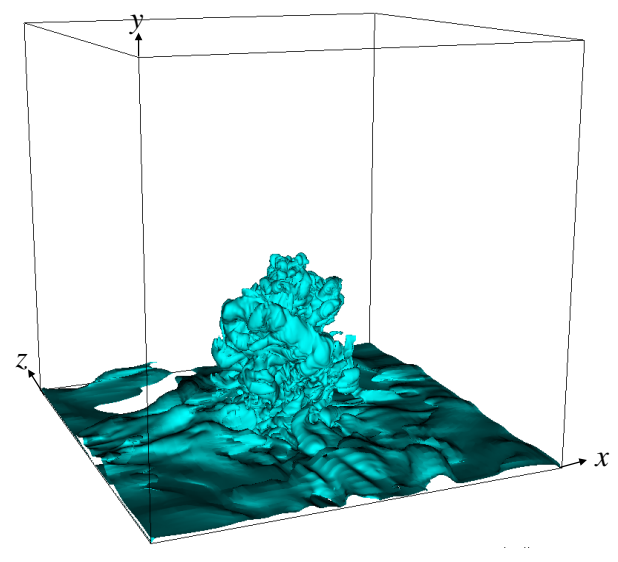

(b)

Figure 1: (a) A schematic of the fountain structure; (b) the domain.

\section{Numerical method}

The flow is simulated by solving the Navier-Stokes equations for incompressible flow, with the Oberbeck-Boussinesq approximation for buoyancy [7]. The dimensionless continuity, momentum, and scalar transport equations are

$$
\begin{aligned}
\frac{\partial u_{i}}{\partial x_{i}} & =0 \\
\frac{\partial u_{i}}{\partial t}+\frac{\partial\left(u_{i} u_{j}\right)}{\partial x_{j}} & =-\frac{\partial p}{\partial x_{i}}+\frac{1}{\operatorname{Re}} \frac{\partial^{2} u_{i}}{\partial x_{j} \partial x_{j}}-\frac{\delta_{i 2} \phi}{\operatorname{Fr}^{2}} \\
\frac{\partial \phi}{\partial t}+\frac{\partial\left(u_{j} \phi\right)}{\partial x_{j}} & =\frac{1}{\operatorname{Re} \operatorname{Pr}} \frac{\partial^{2} \phi}{\partial x_{j} \partial x_{j}},
\end{aligned}
$$

where $\operatorname{Re}=V_{\mathrm{o}} R_{\mathrm{o}} / v$ is the Reynolds number, $\operatorname{Pr}=v / \alpha_{\mathrm{k}}$ is the Prandtl number, and $\mathrm{Fr}=\mathrm{V}_{\mathrm{o}} /\left(\mathrm{g}_{\mathrm{o}} \mathrm{R}_{0}\right)^{0.5}$ is the Froude number, all based on the dimensional source properties; velocity $V_{o}$, temperature $\theta_{0}$, radius $R_{o}$, kinematic viscosity $v$, thermal diffusivity $\alpha_{k}$ and reduced gravity $g_{o}=g\left(\rho_{o}-\rho_{\infty}\right) / \rho_{\infty}$, with $\rho$ 
the density. The $o$ and $\infty$ subscripts indicate properties at the fountain source and in the ambient fluid, respectively. The dimensional velocity $\mathrm{U}_{i}$, temperature $\theta$, pressure $P$, time $T$ and length $X_{i}$ are normalised as $u_{i}=U_{i} / V_{o}$, $\phi=\left(\theta-\theta_{\infty}\right) /\left(\theta_{o}-\theta_{\infty}\right), p=P /\left(\rho V_{o}^{2}\right), t=\mathrm{TV}_{\mathrm{o}} / R_{\mathrm{o}}$ and $x_{i}=X_{i} / R_{o}$. The $i$ subscript for $u_{i}$ represents the three components of velocity $u_{1}=u, u_{2}=v$, $u_{3}=w$ and for $x_{i}$ represents the three dimensions in space $x_{1}=x, x_{2}=y$, $x_{3}=z$, with $y$ vertical (Figure $1(b)$ ). The Einstein summation convention applies for terms with repeated indices, and $\delta_{\mathfrak{i} 2}$ is the Kronecker delta.

The governing equations are discretised by implementing the finite-volume approach on a non-staggered Cartesian grid [13] with standard second order discretisations for all spatial terms other than scalar advectionn for which the ULTRA-QUICK scheme is used [9]. The transport equations are integrated in time using the second-order Adams-Bashforth scheme for the advective terms and the Crank-Nicolson scheme for the viscous and diffusive terms, with the pressure-correction approach used to enforce continuity and obtain pressure. A Jacobi solver is used to invert the transport equations, and a generalized minimum residual (GMRES) method to invert the pressure correction equation, both with Stone's strongly implicit procedure (SIP) pre-conditioning.

As illustrated in Figure 1(b), the computational domain is a rectangular box where the top and side boundaries are open with zero normal-gradient boundary conditions on the velocity and scalar fields. The bottom boundary is a wall with no-slip and adiabatic conditions with normal velocity and temperature, $v=1$ and $\phi=-1$, except for the circular fountain source of radius $r=1$.

The numerical results were obtained on computer clusters for fully developed forced turbulent fountain flows with Reynolds number $2000 \leqslant R e \leqslant 3500$ and Froude number $5 \leqslant \mathrm{Fr} \leqslant 24$. Table 1 gives the details of each of the simulation parameters for each run. Table 1 also gives the grid sizes $\Delta x$ and $\Delta y$ in the central region of the domain, and all $z$ parameters are equal to $x$ parameters. Outside the central region the grid is stretched towards the boundaries. The time step used for all simulations was set to ensure 
Table 1: $\Delta x=\Delta z, \Delta y$ are the finest grid size in the central region of the domain and $\mathrm{L}_{x}=\mathrm{L}_{z}, \mathrm{~L}_{y}, \mathrm{~N}_{x}=\mathrm{N}_{z}, \mathrm{~N}_{y}$ are the domain size and number of nodes in each direction, respectively, $\mathbf{N}_{p}$ is the number of central processing unit (CPU) cores and $t_{\mathrm{cpu}}$ and $t_{\mathrm{s}}$ are the CPU hours and simulation times in non-dimensional units, respectively.

\begin{tabular}{rrrrrrrrr} 
Fr & $\operatorname{Re}$ & $\operatorname{Pr}$ & $\Delta x, \Delta y$ & $\mathrm{~L}_{x}, \mathrm{~L}_{y}$ & $\mathrm{~N}_{x}, \mathrm{~N}_{y}$ & $\mathrm{~N}_{\mathrm{p}}$ & $\mathrm{t}_{\mathrm{cpu}}$ & $\mathrm{t}_{\mathrm{s}}$ \\
\hline 5 & 2500 & 1 & $0.04,0.08$ & 30,30 & 236,265 & 24 & 4332 & 4000 \\
5 & 3000 & 1 & $0.04,0.08$ & 30,30 & 236,265 & 24 & 4332 & 4000 \\
8 & 2500 & 1 & $0.04,0.08$ & 30,40 & 266,350 & 32 & 9272 & 5000 \\
8 & 3000 & 1 & $0.04,0.08$ & 30,40 & 266,350 & 32 & 9272 & 5000 \\
10 & 3500 & 1 & $0.04,0.08$ & 50,50 & 300,400 & 32 & 12488 & 4000 \\
12 & 2000 & 1 & $0.04,0.08$ & 50,60 & 330,503 & 96 & 18624 & 4000 \\
12 & 2750 & 1 & $0.04,0.08$ & 50,60 & 330,503 & 96 & 18624 & 4000 \\
12 & 3500 & 1 & $0.04,0.08$ & 50,60 & 330,503 & 96 & 18624 & 4000 \\
14 & 3000 & 1 & $0.04,0.08$ & 60,80 & 364,550 & 96 & 24456 & 5000 \\
16 & 3500 & 1 & $0.04,0.08$ & 60,80 & 394,675 & 96 & 43320 & 6000 \\
20 & 3000 & 1 & $0.04,0.08$ & 60,110 & 436,800 & 96 & 81216 & 7000 \\
24 & 3000 & 1 & $0.04,0.08$ & 80,120 & 486,975 & 96 & 106752 & 8000
\end{tabular}

the Courant number lay between 0.25 and 0.35 . Grid dependency tests were performed for the highest Reynolds number resulting in a variation of $2 \%$ in fountain height between the fine and coarser grids. The domain is sufficiently large to ensure it does not influence the flow.

\section{Results}

Results were obtained for a range of fully developed fountains as given in Table 1. Figure 1 shows the domain and a single instantaneous temperature contour demonstrating the complex and highly unsteady nature of the fountain flow. The time average results were obtained over a sufficiently long interval 
after the flow reached full development. Time average quantities are shown with an overbar.

Figures 2, 3 and 4 plot the $\bar{\phi}=-0.025$ temperature contour and $\bar{v}=0$ velocity contour. The time-averaged temperature and velocity contours are axisymmetric and plotted against the radius $r$. The $\bar{\phi}=-0.025$ temperature contour is defined to be the boundary between the falling flow and the ambient fluid, whereas the $\bar{v}=0$ velocity contour is the boundary between the rising flow and falling flow. Figures 2(a) and 3(a) show that the fountain height and width have a strong dependence on Fr, with both increasing with increasing Fr. Figures 2(b) and 3(b) show that the dependence is well approximated by a linear scaling with Fr. Figure 4 shows that for $\mathrm{Fr}=12$ there are only relatively small differences between $\mathrm{Re}=2000,2750,3500$ at the boundary of the rising flow and falling flow as well as the boundary of the falling flow and the ambient fluid.

Linear regression is used to obtain the best linear fit for the mean fountain penetration height and width, as shown in Figure 5. The height $h$ is measured vertically on the vertical centreline from the source to the point at which $\bar{\phi}=-0.025$. The width $\mathrm{b}$ is measured radially from the vertical centreline at the mid-height of the fountain to where $\bar{\phi}=-0.025$. The measurements are normalised by the source radius $R_{o}$ and plotted against Fr, giving

$$
\frac{\mathrm{h}}{\mathrm{R}_{\mathrm{o}}}=2.4891 \mathrm{Fr} \quad \text { and } \quad \frac{\mathrm{b}}{\mathrm{R}_{\mathrm{o}}}=0.8824 \mathrm{Fr} \text {. }
$$

Figure 6(a) plots the time-averaged entrainment rate $\bar{\alpha}$ against $y$ for entrainment between the falling flow and ambient fluid on the $\bar{\phi}=-0.025$ contour. The entrainment $\bar{\alpha}=\bar{u}_{r} / V$ where $\bar{u}_{r}$ is the time-averaged radial velocity at $\bar{\phi}=-0.025$ and the bulk falling flow velocity

$$
\mathrm{V}=\left(2 \int_{\mathrm{r} 1}^{\mathrm{r} 2} \overline{v_{\mathrm{y}}}{ }^{2} \mathrm{r} \mathrm{dr}\right)\left(2 \int_{\mathrm{r} 1}^{\mathrm{r} 2} \overline{v_{\mathrm{y}}} \mathrm{r} \mathrm{dr}\right)^{-1}
$$

with $r_{1}$ the radius of the boundary between rising flow and falling flow and $r_{2}$ the radius of the $\bar{\phi}=-0.025$ contour. Away from the crown and base regions, 




(a)

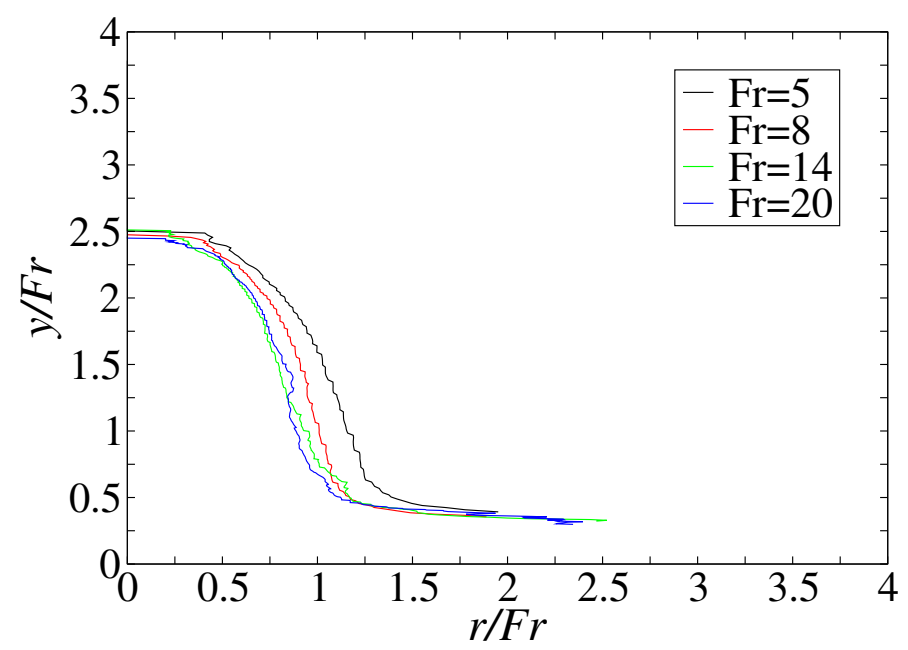

(b)

Figure 2: General structure of forced turbulent fountains for $\mathrm{Fr}=5,8,14,20$ and $\operatorname{Re}=3000$ at: (a) the $\bar{\phi}=-0.025$ temperature contour at the boundary between the falling flow and the ambient fluid; and (b) the $\bar{\phi}=-0.025$ temperature contour scaling with the Froude number. 


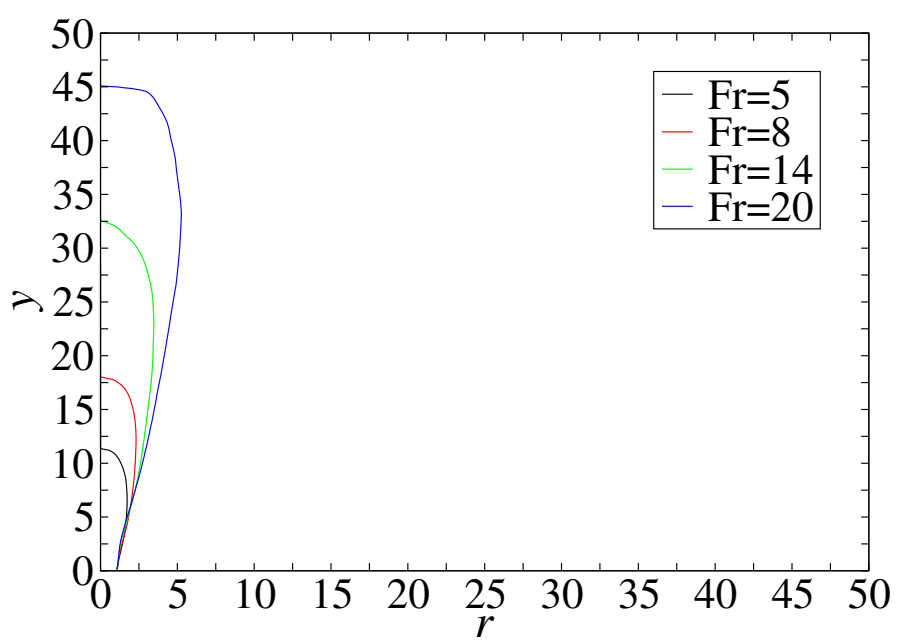

(a)

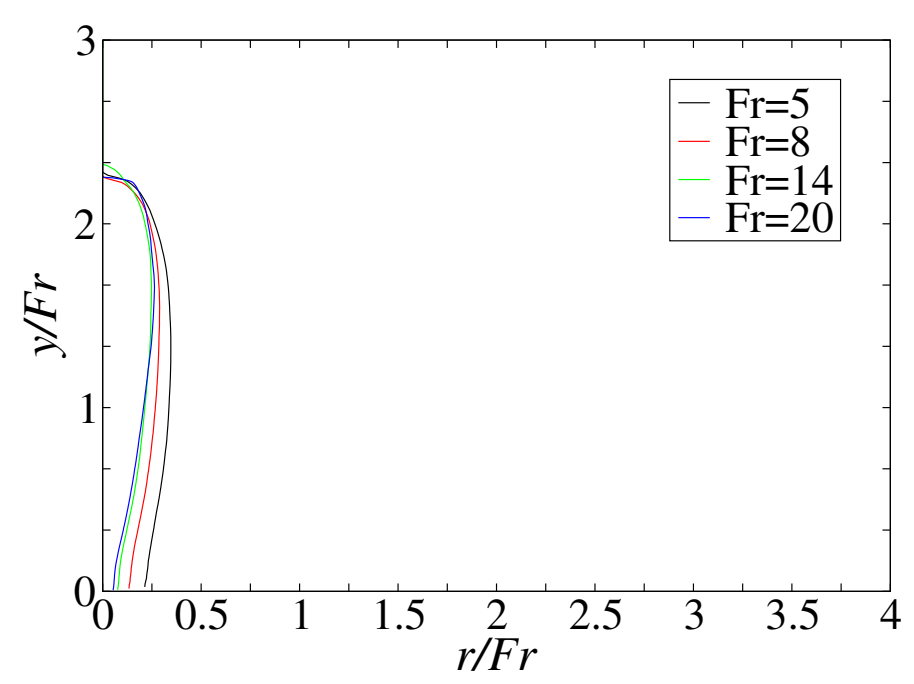

(b)

Figure 3: General structure of forced turbulent fountains for $\mathrm{Fr}=5,8,14,20$ and $\operatorname{Re}=3000$ at (a) the $\bar{v}=0$ velocity contour at the boundary between the rising flow and the falling flow; and (b) the $\bar{v}=0$ velocity contour scaling with the Froude number. 


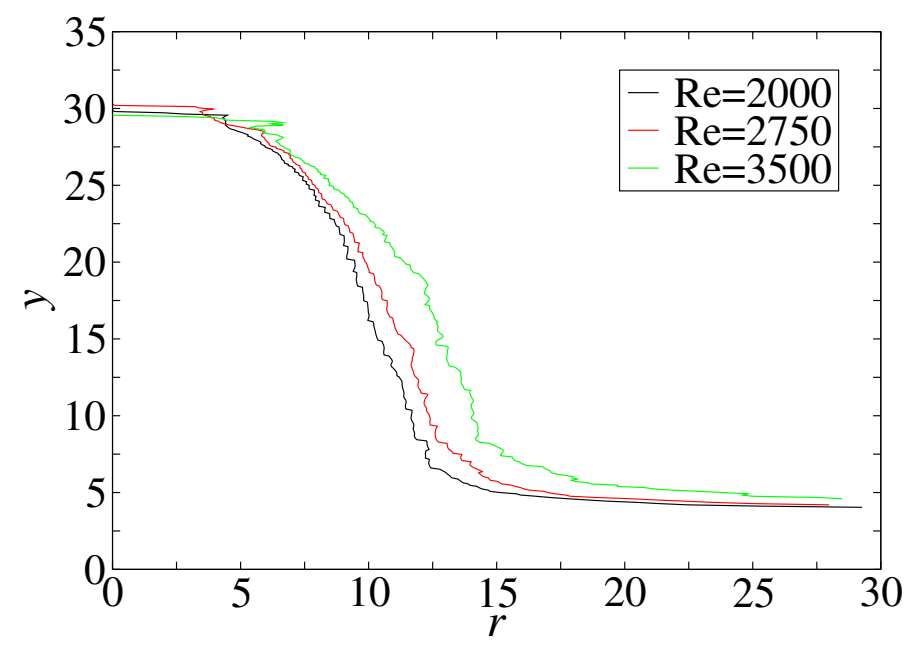

(a)

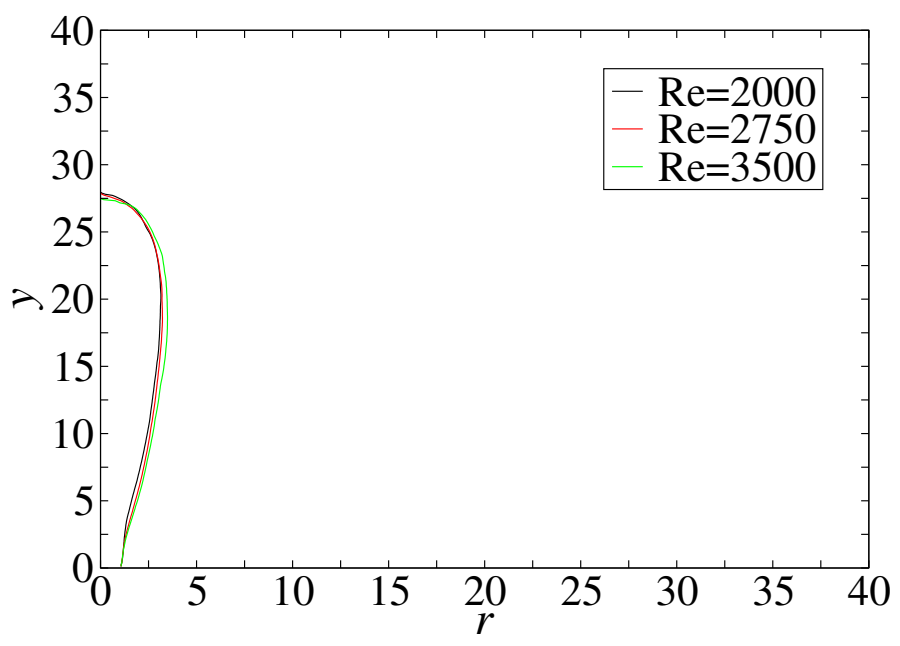

(b)

Figure 4: General structure of forced turbulent fountains for $\operatorname{Re}=$ 2000, 2750, 3500 and Fr $=12$ at: (a) the $\bar{\phi}=-0.025$ temperature contour at the boundary between the falling flow and the ambient fluid; and (b) the $\bar{v}=0$ velocity contour at the boundary between the rising flow and the falling flow. 


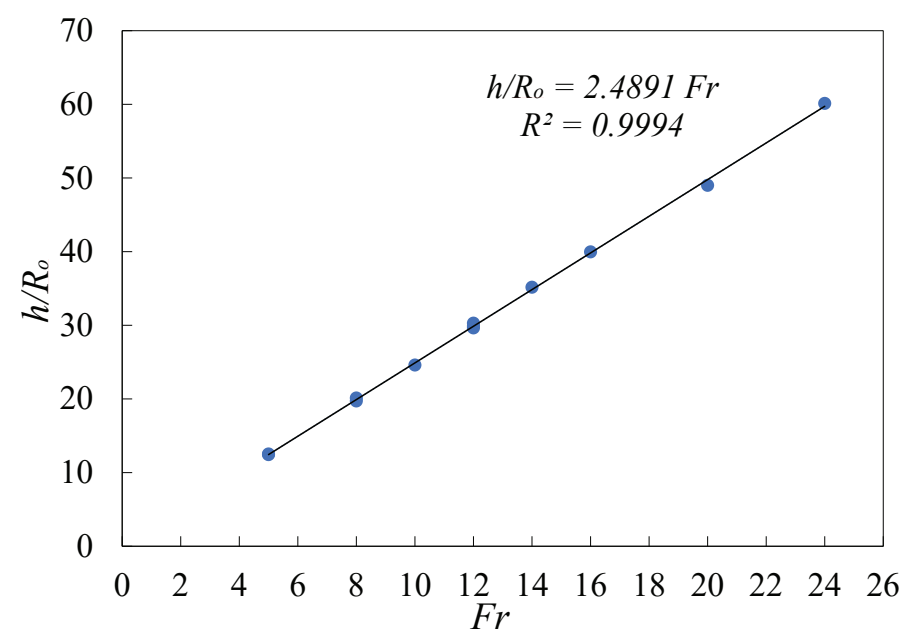

(a)



(b)

Figure 5: Time-averaged scales of forced turbulent fountains with the Froude number for: (a) the mean penetration height scaling with Froude number; and (b) the width scaling with the Froude number. Here, $\mathrm{R}^{2}$ is the linear fit regression coefficient. 


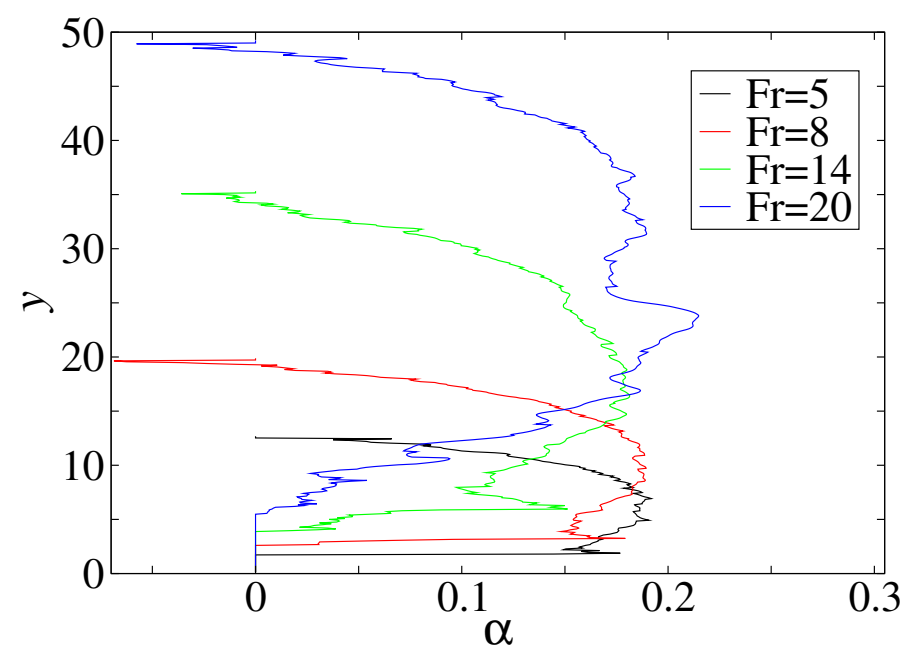

(a)



(b)

Figure 6: Time-averaged entrainment rate of forced turbulent fountains with $\mathrm{Fr}=5,8,14,20$ and $\operatorname{Re}=3000$ : (a) at the boundary between the falling flow and the ambient fluid; and (b) with y scaled by the Froude number. 
$\bar{\alpha}$ is seen to vary between approximately 0.1 and 0.2 . With the vertical axis scaled by Fr, in Figure 6(b), the entrainment is seen to be consistent in the region $0.6 \lesssim y / \operatorname{Fr} \lesssim 2.1$, which represents approximately $60 \%$ of the scaled height. As before, for $\operatorname{Re}=2000,2750,3500$ and $\mathrm{Fr}=12$, the dependency of the entrainment rate on Re is minimal (not shown for brevity).

\section{Conclusions}

Fountains have a rising central flow, a falling outer flow, and a crown region in which the rising flow transitions to the falling flow. The boundary between the rising and falling flows is the zero time-averaged vertical velocity contour. The total fountain height and width was determined by the $\bar{\phi}=-0.025$ temperature contour. The Fr variation of the fountain height and width were shown to be well approximated by linear relations obtained by regression. The scaling for the fountain height corresponds well with previous results [2, $5,11,14,16]$.

The rate of entrainment from the ambient into the falling, outer, fountain fluid was obtained as the radial velocity at the $\bar{\phi}=-0.025$ contour over the bulk vertical velocity in the falling flow region. When the vertical coordinate is scaled with Fr, as for the fountain height, the entrainment coefficients for the different Fr are approximately the same in the region $0.6 \lesssim y / F r \lesssim 2.1$. Over this region $\bar{\alpha}$ lies approximately between 0.1 and 0.2 , compared to the values of $\alpha_{j} \approx 0.075$ for a jet and $\alpha_{p} \approx 0.11$ for a plume.

Acknowledgements: The support of the Australian Research Council is acknowledged through grant DP160102134. 


\section{References}

[1] G. Abraham. "Jets with negative buoyancy in homogeneous fluid". In: J. Hydraul. Res. 5.4 (1967), pp. 235-248. DOI: 10.1080/00221686709500209 (cit. on p. C158).

[2] W. D. Baines, J. S. Turner, and I. H. Campbell. "Turbulent fountains in an open chamber". In: J. Fluid Mech. 212 (1990), pp. 557-592. DOI: 10.1017/S0022112090002099 (cit. on pp. C157, C158, C168).

[3] L. J Bloomfield and R. C Kerr. "A theoretical model of a turbulent fountain". In: J. Fluid Mech. 424 (2000), pp. 197-216. DOI: 10.1017/S0022112000001907 (cit. on p. C158).

[4] H. C. Burridge and G. R. Hunt. "Entrainment by turbulent fountains". In: J. Fluid Mech. 790 (2016), pp. 407-418. DOI: 10.1017/jfm.2016.16 (cit. on p. C158).

[5] I. H. Campbell and J. S. Turner. "Fountains in magma chambers". In: J. Petrol. 30.4 (1989), pp. 885-923. DOI: 10.1093/petrology/30.4.885 (cit. on pp. C158, C168).

[6] P. D. Friedman, V. D. Vadakoot, W. J. Meyer, and S. Carey. "Instability threshold of a negatively buoyant fountain". In: Exp. Fluids 42.5 (2007), pp. 751-759. DOI: 10.1007/s00348-007-0283-5 (cit. on p. C157).

[7] D. D. Gray and A. Giorgini. "The validity of the Boussinesq approximation for liquids and gases". In: Int. J. Heat Mass Tran. 19.5 (1976), pp. 545-551. DOI: 10.1016/0017-9310(76)90168-X (cit. on p. C159).

[8] N. B. Kaye and G. R. Hunt. "Weak fountains". In: J. Fluid Mech. 558 (2006), pp. 319-328. DOI: 10.1017/S0022112006000383 (cit. on pp. C157, C158). 
[9] B. P. Leonard and S. Mokhtari. "Beyond first-order upwinding: The ultra-sharp alternative for non-oscillatory steady-state simulation of convection". In: Int. J. Numer. Meth. Eng. 30.4 (1990), pp. 729-766. DOI: 10.1002/nme.1620300412 (cit. on p. C160).

[10] T. J. McDougall. "Negatively buoyant vertical jets". In: Tellus 33.3 (1981), pp. 313-320. DOI: 10.3402/tellusa.v33i3.10718 (cit. on p. C157).

[11] T. Mizushina, F. Ogino, H. Takeuchi, and H. Ikawa. "An experimental study of vertical turbulent jet with negative buoyancy". In: Wärme Stoffübertrag. 16.1 (1982), pp. 15-21. DOI: 10.1007/BF01322802 (cit. on pp. C158, C168).

[12] B. R. Morton. "Forced plumes". In: J. Fluid Mech. 5.1 (1959), pp. 151-163. DOI: 10.1017/S002211205900012X (cit. on p. C158).

[13] S. E. Norris. "A parallel Navier-Stokes solver for natural convection and free surface flow". PhD thesis. University of Sydney, 2000. DOI: 10.13140/RG.2.1.5072.4964 (cit. on p. C160).

[14] J. S. Turner. "Jets and plumes with negative or reversing buoyancy". In:

J. Fluid Mech. 26.4 (1966), pp. 779-792. DOI:

10.1017/S0022112066001526 (cit. on pp. C158, C168).

[15] N. Williamson, S. W. Armfield, and W. Lin. "Forced turbulent fountain flow behaviour". In: J. Fluid Mech. 671 (2011), pp. 535-558. DOI: 10.1017/S0022112010005872 (cit. on p. C158).

[16] N. Williamson, N. Srinarayana, S. W. Armfield, G. D. McBain, and W. Lin. "Low-Reynolds-number fountain behaviour". In: J. Fluid Mech. 608 (2008), pp. 297-317. DOI: 10.1017/S0022112008002310 (cit. on pp. C157, C158, C168).

[17] H. Zhang and R. E. Baddour. "Maximum penetration of vertical round dense jets at small and large Froude numbers". In: J. Hydraul. Eng. 124.5 (1998), pp. 550-553. DOI:

10.1061/(ASCE) 0733-9429(1998)124:5(550) (cit. on p. C158). 


\section{Author addresses}

1. L. A. Awin, School of Aerospace, Mechanical and Mechatronic Engineering, The University of Sydney, New South Wales 2006, Australia.

mailto:lawi8820@uni.sydney.edu.au

2. S. W. Armfield, School of Aerospace, Mechanical and Mechatronic Engineering, The University of Sydney, New South Wales 2006, Australia.

3. N. Williamson, School of Aerospace, Mechanical and Mechatronic Engineering, The University of Sydney, New South Wales 2006, Australia.

4. M. P. Kirkpatrick, School of Aerospace, Mechanical and Mechatronic Engineering, The University of Sydney, New South Wales 2006, Australia.

5. W. Lin, College of Science and Engineering, James Cook University, Queensland 4811, Australia. 